\title{
Instability and "Sausage-String” Appearance in Blood Vessels during High Blood Pressure
}

\author{
Preben Alstrøm, ${ }^{1}$ Victor M. Eguíluz, ${ }^{2}$ Morten Colding-Jørgensen, ${ }^{3}$ \\ Finn Gustafsson, ${ }^{4}$ and Niels-Henrik Holstein-Rathlou ${ }^{4}$ \\ ${ }^{1}$ CATS, The Niels Bohr Institute, DK-2100 Copenhagen, Denmark \\ ${ }^{2}$ Instituto Mediterráneo de Estudios Avanzados IMEDEA (CSIC-UIB), E-07071 Palma de Mallorca, Spain \\ ${ }^{3}$ Novo Nordisk, DK-2880 Bagsvaerd, Denmark \\ ${ }^{4}$ Department of Medical Physiology, The Panum Institute, DK-2200 Copenhagen, Denmark
}

(Received 1 October 1998)

\begin{abstract}
A new Rayleigh-type instability is proposed to explain the "sausage-string" pattern of alternating constrictions and dilatations formed in blood vessels under influence of a vasoconstricting agent. Our theory involves the nonlinear elasticity characteristics of the vessel wall, and provides predictions for the conditions under which the cylindrical form of a blood vessel becomes unstable. [S0031-9007(99)08585-3]

PACS numbers: 87.19.Rr, 02.30.Jr, 47.20.Dr
\end{abstract}

High blood pressure can experimentally be induced by intravenous infusion of a vasoconstricting agent such as angiotensin II (which regulates the contraction of the smooth muscle cells surrounding the blood vessel) [1-3]. As the infusion is continued, a substantial narrowing of the smaller blood vessels is observed, and suddenly the narrowed vessels develop a peculiar pattern consisting of alternating regions of constrictions and dilatations, giving the vessels the appearance of sausages on a string (Fig. 1). The "sausage-string" pattern may cause severe damage to the blood vessels because plasma and macromolecules are transported into the vessel wall in the dilated regions. The sausage-string pattern has been observed in small vessels from many organs, including the brain, the gut, and the kidney [4].

Despite several decades of research, the mechanism causing the sausage-string pattern has remained unknown [4]. It has been suggested that it represents a "blowout" of the vessel wall due to the high blood pressure [5], but this seems unlikely for several reasons. The sausage-string pattern occurs in the smaller vessels (small arteries and large arterioles), and here the pressure elevation is relatively small compared to that in the larger arteries. Second, the phenomenon is highly reproducible [2]. If the infusion of the vasoconstricting agent is stopped, the normal, uniform "cylindrical" structure is restored. Restoring the infusion causes again an extreme, uniform vasoconstriction followed by the reappearance of the sausage-string pattern. A third spectacular feature of the phenomenon is its periodicity with constrictions and dilatations occurring in a regular and repetitive pattern.

In this Letter, we present a simple anisotropic, elastic model of the vessel wall. We show that under certain hypertensive conditions an instability occurs which leads to a periodic pattern of constrictions and dilatations along the vessel. Our theory provides predictions for the conditions under which the cylindrical form of a blood vessel becomes unstable.
To be specific, a cylindrical shaped blood vessel is unstable if a small axial symmetric perturbation of the inner radius, $r \rightarrow r+u(z)$, grows (Fig. 2). To determine the stability, we must therefore know the dynamic equation for the perturbation $u(z, t)$. To this end, we invoke the continuity equation, $\partial_{t}\left(\pi r^{2}\right)=-\partial_{z} J$, associating a local change of the cross-sectional area at a downstream site $z$ with a fluid flux $J(z)$. The flux is related to the transmural pressure $P$, by $J=-c(r) \partial_{z} P$, where $c(r)$ is the vascular conductance [6]. From the continuity equation and the flux-pressure relation, the dynamic equation to lowest order in the perturbation follows:

$$
\partial_{t} u=\frac{c(r)}{2 \pi r} \partial_{z}^{2} P .
$$

As a simple illustration, consider first a very thin vessel wall, for which the pressure is given by the Laplace form

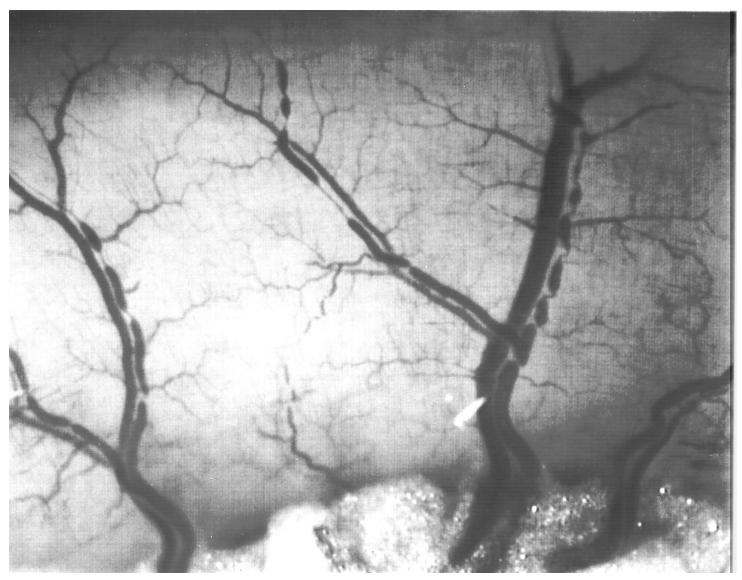

FIG. 1. In vivo micrograph of rat intestinal arterioles showing a typical "sausage-string" pattern following an acute increase in blood pressure induced by intravenous infusion of angiotensin II. The neighboring vessels not showing constrictions and dilatations are the corresponding venules. From [4] with permission. 

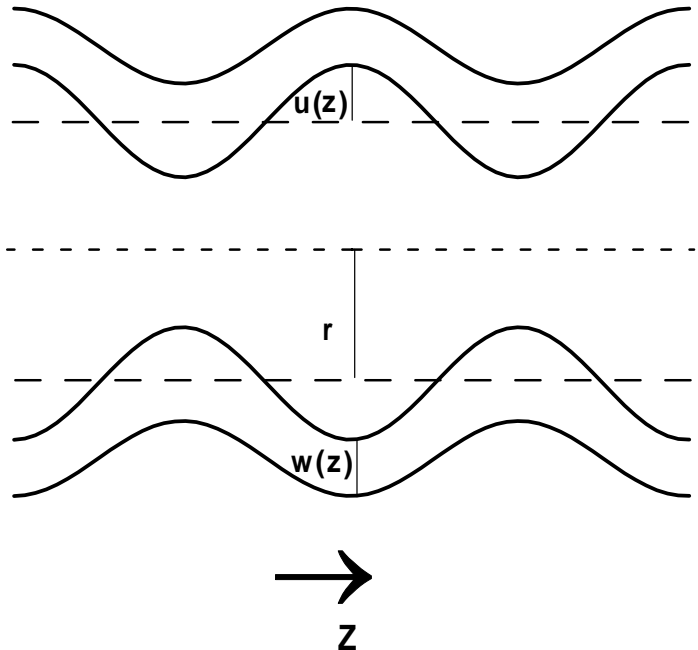

FIG. 2. A schematic picture of a blood vessel of inner radius $r$ undergoing a perturbation $u(z)$. The wall thickness $w(z)$ is larger at smaller radii since the circumference is smaller.

[7,8] $P=(T / R)+\left(T_{z} / R_{z}\right)$, where $T$ and $T_{z}$ are the tensions in the principal angular and vessel directions, and $1 / R$ and $1 / R_{z}$ are the corresponding curvatures [9]. Assume furthermore that the tensions are constant and identical, $T_{z}=T$. Inserting the above expression for the pressure into Eq. (1) and retaining only first order terms in $u$, we get

$$
\partial_{t} u=-\frac{T c(r)}{2 \pi r^{3}}\left[\partial_{z}^{2} u+r^{2} \partial_{z}^{4} u\right] .
$$

For a given periodic perturbation, $u=u_{k}(t) \cos (k z)$, we have $u_{k}(t) \sim u_{k}(0) e^{\lambda_{k} t}$, where

$$
\lambda_{k}=\frac{T c(r)}{2 \pi r^{3}} k^{2}\left[1-r^{2} k^{2}\right] \text {. }
$$

Thus, the vessel wall is unstable to modes with $r k<1$. The dominant mode, where $\lambda_{k}$ is maximal, is at $k=1 / \sqrt{2} r$.

The above instability is the well-known Rayleigh instability $[10,11]$. The theory explains why a cylindrical column of water with surface tension $T$ is unstable at all radii. However, cylindrical structures may be stable due to a reluctance against bending [12]. Still an instability may occur if the tension $T$ can be brought to exceed a critical value of order $\kappa / r^{2}, \kappa$ being the bending modulus [12]. This is demonstrated by the so-called "pearling" instability, recently observed by Bar-Ziv and Moses [13] in tubular lipid membranes.

For blood vessels the width $w$ of the vessel wall cannot be neglected. Here, the vessel wall is viewed as a layered structure, where the only stresses are in the principal angular and vessel directions [8]. There are no shear stresses, and the stress in the direction normal to the layers is zero. With these assumptions, the Laplacian form $[7,8]$ for the pressure is replaced by an integral [14,15],

$$
\begin{aligned}
P=\int_{r}^{r+w} & {\left[S \frac{1}{\tilde{r}\left[1+\left(\partial_{z} \tilde{r}\right)^{2}\right]^{1 / 2}}\right.} \\
& \left.-S_{z} \frac{\partial_{z}^{2} \tilde{r}}{\left[1+\left(\partial_{z} \tilde{r}\right)^{2}\right]^{3 / 2}}\right] d \tilde{r},
\end{aligned}
$$

where $S$ and $S_{z}$ are the stresses in the angular and vessel directions. The stresses, defined as the forces per actual cross-sectional area, are related to the experimentally measured idealized stresses $\sigma$ and $\sigma_{z}$, defined as the forces per relaxed cross-sectional area, $S=\gamma \gamma_{z} \sigma$ and $S_{z}=\gamma \gamma_{z} \sigma_{z}$ [15]. Here, $\gamma$ and $\gamma_{z}$ are the normalized lengths [16] in the angular and vessel direction. The length of a vessel remains almost constant during a contraction, and $\gamma_{z}$ is therefore assumed to be constant, $\gamma_{z}=\gamma_{0}$. Correspondingly, the stress $\sigma_{z}$ is also replaced by a constant $\sigma_{0}$. We note that the width $w$ of the vessel wall changes when the inner radius $r$ changes (Fig. 2). Assuming that the crosssectional area of the vessel wall is constant, the (inner) radius dependence of $w$ is given, when the inner radius $\rho$ and wall thickness $\omega$ are known for the relaxed vessel $(\gamma=1)[15,17]$.

For small perturbations, the relevant expression for the pressure reduces to

$$
P=\gamma_{0} \int_{\rho}^{\rho+\omega}\left[\sigma-\sigma_{0} r \partial_{z}^{2} r\right]\left[\tilde{\rho}^{2}-\rho^{2}+r^{2}\right]^{-1 / 2} d \tilde{\rho} .
$$

For the angular direction, the stress $\sigma$ depends on the normalized length $\gamma=\left[\tilde{\rho}^{2}-\rho^{2}+r^{2}\right]^{1 / 2} / \tilde{\rho}$. To first order in the perturbation $u(z, t)$, we find

$$
P=P_{0}(r)+I(r) u-I_{0}(r) \partial_{z}^{2} u,
$$

where

$$
\begin{aligned}
P_{0}(r) & =\gamma_{0} \int_{\rho}^{\rho+\omega} \sigma\left[\tilde{\rho}^{2}-\rho^{2}+r^{2}\right]^{-1 / 2} d \tilde{\rho}, \\
I_{0}(r) & =\gamma_{0} \sigma_{0} r \int_{\rho}^{\rho+\omega}\left[\tilde{\rho}^{2}-\rho^{2}+r^{2}\right]^{-1 / 2} d \tilde{\rho} \\
& =\gamma_{0} \sigma_{0} r \log [1+(\omega+w) /(\rho+r)],
\end{aligned}
$$

and

$$
I(r)=\frac{d}{d r} P_{0}(r)=\gamma_{0} \int_{\rho}^{\rho+\omega} \tilde{\rho}^{-1} \frac{d}{d \gamma}\left[\frac{\sigma}{\gamma}\right] \frac{\partial \gamma}{\partial r} d \tilde{\rho} .
$$

The partial derivatives of $\gamma$ with respect to $r$ and $\tilde{\rho}$ are related, $\tilde{\rho}^{-1}(\partial \gamma / \partial r)=r\left(\rho^{2}-r^{2}\right)^{-1}(\partial \gamma / \partial \tilde{\rho})$, and $I(r)$ can be expressed in terms of the normalized length $\gamma$,

$$
I(r)=\frac{\gamma_{0} \gamma_{r}}{\rho\left(1-\gamma_{r}^{2}\right)}\left[\frac{\sigma\left(\gamma_{w}\right)}{\gamma_{w}}-\frac{\sigma\left(\gamma_{r}\right)}{\gamma_{r}}\right],
$$


where $\gamma_{r}=r / \rho$ is the normalized inner radius, and $\gamma_{w}=(r+w) /(\rho+\omega)$ is the normalized outer radius. We note that $I(r)$ is not singular at $\gamma_{r}=1$, where also $\gamma_{w}=1$. Inserting Eq. (6) into Eq. (1) we get for a given periodic perturbation, $u=u_{k}(t) \cos (k z)$, that $u_{k}(t) \sim$ $u_{k}(0) e^{\lambda_{k} t}$, where

$$
\lambda_{k}=\frac{c(r)}{2 \pi r} k^{2}\left[-I(r)-I_{0}(r) k^{2}\right] .
$$

The value of $I_{0}(r)$ is always positive. Thus, it is the sign of $I(r)$ that determines the stability of the vessel wall. If $I(r)$ is positive, the cylindrical shape is stable for all modes. If $I(r)$ is negative, the cylindrical shape is unstable to modes with $k^{2}<|I| / I_{0}$.

As seen from the expression for $I$, Eq. (10), the important quantity is $\sigma / \gamma$. The point of instability is where $\sigma / \gamma$ calculated at the inner radius equals the value of $\sigma / \gamma$ at the outer radius. This can be illustrated geometrically by drawing a line in the plot of $\sigma$ versus $\gamma$ (Fig. 3) from $(0,0)$ through $\left(\gamma_{r}, \sigma\left(\gamma_{r}\right)\right)$. If the point $\left(\gamma_{w}, \sigma\left(\gamma_{w}\right)\right)$ lies above this line, $I(r)$ is positive and the cylindrical form is stable. If, however, the point $\left(\gamma_{w}, \sigma\left(\gamma_{w}\right)\right)$ lies below this line, $I(r)$ is negative, leading to an instability of the cylindrical form.

Under normal physiological conditions, the angular stress $\sigma$ in blood vessels increases linearly to exponen-

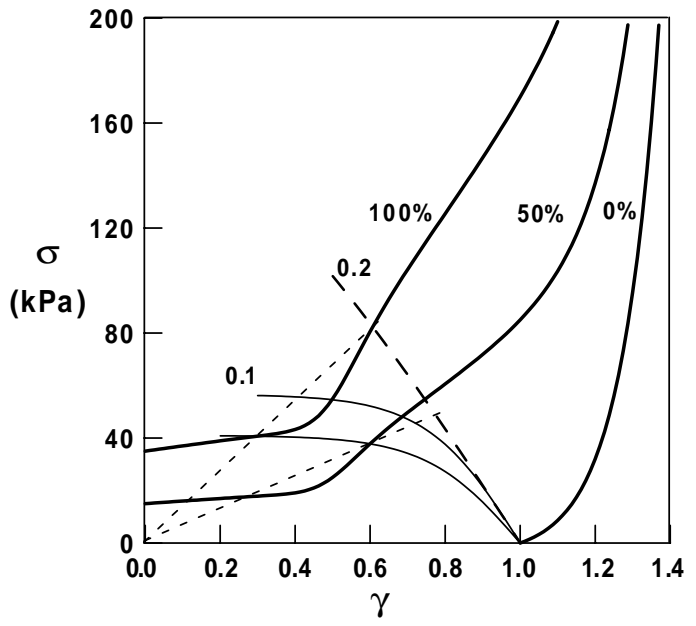

FIG. 3. A schematic plot of typical stress-strain relations for arterioles (adapted from [18]). The three heavy solid curves correspond to a vessel where the smooth muscle cells are not activated $(0 \%)$, where the smooth muscle cells are half maximally activated (50\%), and where the smooth muscle cells are maximally activated $(100 \%)$. The thin solid lines show how the points $\left(\gamma_{r}, \sigma\left(\gamma_{r}\right)\right)$ and $\left(\gamma_{w}, \sigma\left(\gamma_{w}\right)\right)$ [marked $0.1]$ move with muscle cell activation for an arteriole with wall-to-lumen ratio $\omega / \rho=0.1$. The point of instability $\left(r=r_{c}\right)$ for the cylindrical form of the blood vessel is illustrated geometrically by thin dashed lines from $(0,0)$ through $\left(\gamma_{r}, \sigma\left(\gamma_{r}\right)\right)$. The instability point is where $\sigma\left(\gamma_{w}\right) / \gamma_{w}$ equals $\sigma\left(\gamma_{r}\right) / \gamma_{r}$. The thick dashed line [marked 0.2] shows how the point $\left(\gamma_{w}, \sigma\left(\gamma_{w}\right)\right)$ moves with muscle cell activation for an arteriole with $\omega / \rho=0.2$, keeping the same curve for $\left(\gamma_{r}, \sigma\left(\gamma_{r}\right)\right)$. tially with the normalized length $[8,18]$ (Fig. 3), and the value of $I(r)$ is therefore positive (Fig. 4). This ensures that the blood vessel keeps its cylindrical shape-no bending arguments are needed to explain the stability of the cylindrical shape of a blood vessel. However, when acute hypertension is induced by infusion of a strong vasoconstricting agent like angiotensin II, there will be a substantial reduction of the inner radius in small arteries and large arterioles due to contraction of the smooth muscle cells. The operating point for the vessel will now be on a less steep part of the $\sigma-\gamma$ curve (Fig. 3), and, when the inner radius is reduced below a certain value $r_{c}$ at which $\sigma\left(\gamma_{w}\right) / \gamma_{w}=\sigma\left(\gamma_{r}\right) / \gamma_{r}$, the value of $I(r)$ becomes negative (Fig. 4). This will result in an instability of the cylindrical form, giving rise to the sausage-string pattern. The dominant (fastest growing) mode is given by $k=\left[|I| / 2 I_{0}\right]^{1 / 2}$, which will correspond to "sausages" of length $\ell=2 \pi \sqrt{2 I_{0} /|I|}$. Insertion of typical values [19] for the various parameters of the model yields $\ell \approx 2 \pi \rho$; hence, the length of the sausages will be 5-10 times the radius of the relaxed vessel. The theory therefore predicts that the sausages will have an elongated shape with a length that decreases as the vessel radius gets smaller. This is in good agreement with experimental observations [2] (Fig. 1).

A way to view the basic phenomenon underlying the instability is to note that, when $I(r)$ becomes negative, the pressure at slightly larger radii is smaller than at slightly smaller radii. Accordingly, the resulting flow $J$ will be directed from small-radii regions to large-radii regions, causing the small radii to become even smaller, and the large radii to become larger. This continues until the pressure stabilizes at a value which is the same for both the large

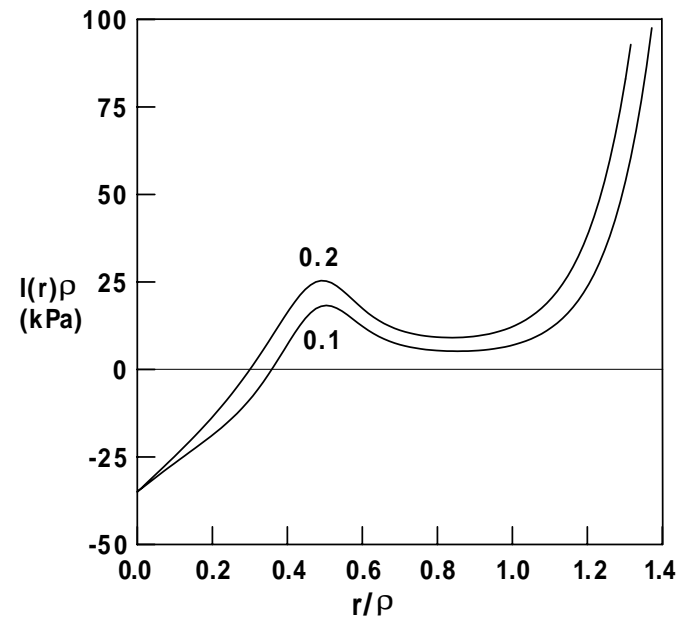

FIG. 4. A plot of the stability measure $I(r)$ at large muscle cell activation for two different wall-to-lumen ratios, $\omega / \rho=$ 0.1 and $\omega / \rho=0.2$. The cylindrical form of a blood vessel becomes unstable when $I$ becomes negative. An almost linear stress-strain relation in a region above $r_{c}$ gives rise to a decay of $I$. Above $\gamma=1$, where the stress increases exponentially, also $I(r)$ increases exponentially. 
radius $r_{\max }$ and the small radius $r_{\min }$. The stabilization is only possible because the pressure for radii above the instability, $r>r_{c}$, again increases with $r$. The theory allows an estimate of the radius in the dilated regions, $r_{\max }$. Assuming that $r_{\min } / \rho$ is small (close to zero), we can estimate the final value of $r_{\max }$ by the condition $P\left(r_{\max }\right)=P(0)$. Interestingly, the almost linear stress function in the region above $r_{c}$ (Fig. 3) gives rise to a decay of $I(r)$ in the same region (Fig. 4). As a consequence $r_{\max }$ can become quite large. However, close to $\gamma=1$, the stress increases exponentially due to the elastic properties of the vessel wall [8], and the value of $I(r)$ will increase rapidly. This will effectively prevent $r_{\max }$ from attaining a value substantially larger than the relaxed vessel radius, $\rho$. However, $r_{\max }$ may be larger than the working radius of the vessel under normal physiological conditions, because the normal working radius is smaller than the relaxed vessel radius [8]. This may explain why previous articles have suggested that the dilated regions represented a blowout due to mechanical failure of the vessel wall [5].

The sausage-string pattern following infusion of angiotensin II have been found to occur predominantly in small arteries and large arterioles [2]. The present analysis predicts that large vessels will be stable. Their operating points are on the steep portion of the $\sigma-\gamma$ curve due to their high pressure. As seen from Fig. 3, the contraction is here limited, thus preventing the larger vessels from reducing their (inner) radius below the critical value $r_{c}$. As arterial vessels get smaller, the wall-to-lumen ratio $\omega / \rho$ increases [8]. From the expression for $I(r)$, Eq. (10), we find that $r_{c}$ as well as $r_{\max }$ decreases with an increasing wall-to-lumen ratio. Hence, the sausage-string instability is less likely to appear in blood vessels with large wallto-lumen ratios. It seems that the transmural pressure and the contractile potential sets an upper limit, and the wallto-lumen ratio a lower limit for vessels that will undergo the sausage-string instability in response to an acute increase in blood pressure.

In summary, we have demonstrated that, during severe vasoconstriction, the normal cylindrical shape of a blood vessel may become unstable, and as a result the vessel exhibit a periodic pattern of constrictions and dilatations. The sausage-string pattern is not caused by a mechanical failure of the vessel wall due to the high blood pressure, but is the expression of an instability. The instability is related to the Rayleigh instability, and to the pearling instability seen in tubular lipid membranes. The mechanism behind the instability, however, is novel, involving the nonlinear elasticity characteristics of the vessel wall. The developed theory explains many of the key features observed experimentally, e.g., the predominant occurrence in small arteries and large arterioles, and most likely in those with small wall-to-lumen ratios.

The present study was supported by grants from the Danish Natural Science Research Council, the Danish
Medical Research Council, the Novo-Nordisk Foundation, and the Danish Heart Association.

[1] F. B. Byrom, Lancet 2, 201 (1954); F. B. Byrom, Prog. Cardiovasc. Dis. 1, 31 (1974).

[2] J. Giese, Acta Pathol. Microbiol. Scand. 62, 497 (1964).

[3] J. Giese, The Pathogenesis of Hypertensive Vascular Disease (Munksgaard, Copenhagen, 1966).

[4] F. Gustafsson, Blood Pressure 6, 71 (1997).

[5] L. J. Beilin and F. S. Goldby, Clin. Sci. Mol. Med. 52, 111 (1977).

[6] In the Hagen-Poiseuille approximation, the fluid conductance is $c(r)=\pi r^{4} / 8 \eta$, where $\eta$ is the dynamic viscosity of the fluid (blood), but the specific form of $c(r)$ is not crucial for our purpose. Moreover, we neglect the pressure drop along the vessel, noting that this is much smaller than the transmural pressure.

[7] A. E. Green and J. E. Adkins, Large Elastic Deformations (Clarendon Press, Oxford, 1960).

[8] Y.C. Fung, Biomechanics. Mechanical Properties of Living Tissues (Springer-Verlag, New York, 1990), 2nd ed.; Y.C. Fung, Biomechanics. Motion, Flow, Stress, and Growth (Springer-Verlag, New York, 1990).

[9] The principal curvatures $1 / R$ and $1 / R_{z}$ are given by the following two relations:

$$
\frac{1}{R}=\frac{1}{r\left[1+\left(\partial_{z} r\right)^{2}\right]^{1 / 2}}, \quad \text { and } \quad \frac{1}{R_{z}}=\frac{-\partial_{z}^{2} r}{\left[1+\left(\partial_{z} r\right)^{2}\right]^{3 / 2}} .
$$

[10] J. Plateau, Statique Experimentale et Theorique des Liquides Soumis aux Seules Forces Moleculaires (GautierVillars, Paris, 1873).

[11] Lord Rayleigh, Philos. Mag. 34, 145 (1892).

[12] P. Nelson and T. Powers, Phys. Rev. Lett. 74, 3384 (1995); R. E. Goldstein, P. Nelson, T. Powers, and U. Seifert, J. Phys. II (France) 6, 767 (1996).

[13] R. Bar-Ziv and E. Moses, Phys. Rev. Lett. 73, 1392 (1994).

[14] R. S. Chadwick, Biophys. J. 39, 279 (1982).

[15] R. Feldberg, M. Colding-Jørgensen, and N.-H. HolsteinRathlou, Am. J. Phys. 269, F581 (1995).

[16] The normalized length $\gamma$ at a given radius $\tilde{r}(r<\tilde{r}<$ $r+w)$ is equal to the ratio between the circumference $2 \pi \tilde{r}$ of the vessel strip situated at the radius $\tilde{r}$ and the corresponding circumference $2 \pi \tilde{\rho}(\rho<\tilde{\rho}<\rho+\omega)$ of the same vessel strip in the relaxed vessel, where the smooth muscle cells are not activated and the transmural pressure is zero. The corresponding strain is by definition equal to $\gamma-1$.

[17] We have $\pi(r+w)^{2}-\pi r^{2}=\pi(\rho+\omega)^{2}-\pi \rho^{2}$. This equation suggests a useful change of variable, from $\tilde{r}$ to $\tilde{\rho}$, where $\tilde{r}^{2}-r^{2}=\tilde{\rho}^{2}-\rho^{2}$. When $\tilde{r}$ varies between values $r$ and $r+w$, which under perturbations changes along the $z$ axis, $\tilde{\rho}$ varies between the fixed inner and outer radii $\rho$ and $\rho+\omega$ of the relaxed vessel. The normalized length $\gamma$ at a radius $\tilde{r}$ is $\gamma=\tilde{r} / \tilde{\rho}$.

[18] R. W. Gore, Circ. Res. 34, 581 (1974); M. J. Davis and R. W. Gore, Am. J. Phys. 256, H630 (1989).

[19] For $\omega / \rho=0.1$, and $\sigma_{0}=100 \mathrm{kPa}$, we have $|I| \rho \approx$ $2 I_{0} / \rho \approx 10 \mathrm{kPa}$. 